\title{
La historia de un legado: la colección bibliográfica MacCormack en el Perú
}

\author{
Fernando Valle Rondón
}

\section{RESUMEN}

Esta breve crónica ofrece un recuento de las circunstancias en que la colección bibliográfica de Sabine MacCormack fue remitida al Perú, donada al Instituto de Pastoral Andina y recibida en custodia por la Universidad San Pablo de Arequipa, donde se aloja temporalmente. Además de algunos detalles referidos a la complejidad del traslado, se ofrecen reflexiones sobre la relación de la autora con el mundo andino y un agradecimiento a las personas que ayudaron a que ese valioso legado llegara al Perú. ${ }^{1}$

1 Esta breve crónica ha sido elaborada en diálogo con mi colega, el profesor César Félix Sánchez Martínez, docente de filosofía, de literatura y miembro de la Sociedad Peruana de Historia, a quien yo le había pedido encarecidamente hacer un artículo sobre el valor de la colección bibliográfica donada por Sabine MacCormack al Instituto de Pastoral Andina, artículo conexo publicado en este mismo número. Dado que yo me había encargado del traslado de la colección MacCormack al Perú, juzgamos conveniente separar en dos textos independien- 
Palabras Clave: Sabine MacCormack, colección bibliográfica, legado, donación internacional

This chronicle shows the circumstances in which Sabine MacCormack's book collection -donated to the Instituto de Pastoral Andina (IPA) and stored in U. Catolica San Pablo in Arequipa- was inventoried, packed and delivered to Peru. The author -who was in charge of these procedures in South Bend MacCormack's house- shares his experiences there, his impressions on her relationship to the Andean world and his gratitude to every person who was involved into this valuable project.

KEYwORDs: Sabine MacCormack, book collection, legacy, international donation

El 4 DE DICIEMBre DE 2012 leí un mensaje de correo electrónico bastante singular que había sido enviado por un señor Thomas Cornfield a la dirección electrónica del Instituto de Pastoral Andina. En ese momento, yo me despeñaba como su director ad interim. El contenido del mensaje era aún más sorprendente: allí, el señor Cornfield se presentaba como ejecutor testamentario o albacea de la recientemente fallecida profesora Sabine MacCormack, de South Bend, Indiana (EUA) y nos comunicaba que su biblioteca había sido donada por testamento a nuestra institución. Nos preguntaba si teníamos a bien recibirla legalmente y transportarla hacia el Perú. Después de varias aceleradas consultas y gestiones internas en el IPA y en la Universidad Católica San Pablo de Arequipa, pude, gracias a Dios, responderle afirmativamente, agradeciendo por supuesto tanta generosidad.

tes el análisis de la colección bibliotecaria, por un lado, y por otro, los detalles de las gestiones de la recepción, inventario, embalaje, transporte y otros detalles que podrían también gozar del interés de nuestros lectores. A él, mi sentido agradecimiento por ayudarme en la elaboración de esta sencilla crónica, tanto por la escucha atenta a los detalles como por las reflexiones historiográficas que surgieron en este fecundo diálogo. 
Yo felizmente conocía la obra de Sabine MacCormack, Religion in the Andes (1991), pues la había leído durante mi periodo universitario, cuando estudiaba historia en la Pontificia Universidad Católica del Perú, hace ya unos 25 años. Dicha obra me impresionó mucho porque ponía de relieve las tendencias filosóficas y teológicas de los autores españoles que buscaban comprender la religión andina. Al mismo tiempo, influidos por un marco cultural claramente cristiano, mostraba cómo los autores andinos trataban de encontrar en dicha religión ancestral los antecedentes de su nueva fe cristiana. MacCormack insistía en que la religión andina debe ser entendida en el marco cultural que influyó y condicionó a sus actores históricos, tanto indios como occidentales. Se refiere a dos tradiciones religiosas opuestas con claros rasgos distintivos aunque en continua transformación, que limitaron y caracterizaron su desarrollo durante unos 130 años, desde la conquista hasta 1660. Este esfuerzo por confrontarse directamente con una religión «colonial» me parecía más realista que la pretensión de ver la religión andina sin los «lentes occidentales», a través de una metodología que pone entre paréntesis las características consideradas no nativas, lo cual no siempre es fácil de percibir, captar, distinguir o separar.

Así, yo sabía de su prestigio como estudiosa del mundo andino, pero no conocía de su colección bibliográfica. Una vez que recibí el mensaje de correo electrónico, realicé algunas indagaciones al respecto y la respuesta fue casi unánime: jera una colección magnífica! En este punto, David Dressing, estudioso y bibliotecario encargado del área de estudios latinoamericanos e ibéricos de la Universidad de Notre Dame (UND) nos ayudó bastante en la primera valoración del legado. No obstante, en realidad nadie sabía a ciencia cierta de cuántos o de cuáles libros estábamos hablando. No existía un inventario de los libros. Y sabíamos que por eso debíamos empezar.

Inmediatamente conversé con el rector de la Universidad Católica San Pablo -socia estratégica permanente y luego miembro 
del comité del Instituto de Pastoral Andina- sobre esta promisoria posibilidad. Quiso la Providencia que nuestro rector, Dr. Germán Chávez Contreras, tuviera programada por aquella misma época una visita académica al Calvin College, en Grand Rapids, ciudad cercana a South Bend, donde se encuentra la casa en la que Sabine había vivido. Más sorprendente aún fue la grata coincidencia en el viaje de ida con un viejo amigo y antiguo profesor de la UCSP, el Dr. Carlos Vargas Salgado, y un becario peruano de la Universidad de Notre Dame, Víctor Maqque, quienes admiraban el trabajo historiográfico de Sabine y tenían muy buenas referencias de su biblioteca. ¡Le hablaron maravillas de la colección!

Con estos datos introductorios, llegó el Dr. Chávez a la casa de la prestigiosa académica el 22 de mayo del 2013 para conocer personalmente la colección de la mano de Catherine MacCormack, la hija de Sabine, encantadora persona que siempre dio todas las facilidades para que se cumpla la voluntad de su madre. A su regreso y después de elogiar la colección donada, Germán me pidió que me encargase de los trámites de recepción y traslado de los libros al Perú. El viaje de Arequipa a South Bend duró 25 horas y me alojé en la misma universidad, que quedaba a tres millas, durante poco más de dos semanas, desde el 14 de julio hasta el primero de agosto. Se me había encargado la enorme tarea de elaborar un inventario básico de todos los libros y después embalarlos y etiquetarlos para su transporte por barco. Debía también encargarme de las gestiones legales y logísticas pertinentes en Estados Unidos, país en el que felizmente hay una mayor eficiencia legal en los procesos de ejecución testamentaria. Afortunadamente tuve, además de la colaboración de entusiastas estudiantes de la UND, la ayuda remota de personal especializado en Arequipa con quien podía coordinar los complejos trámites aduaneros.

Al llegar a la antigua casa de Sabine, quedé impresionado por la magnitud de la empresa. Era una casa americana de tres pisos, todos los cuales contenían libros muy bien conservados, ordenados y bien 
dispuestos. La biblioteca no estaba localizada en un solo ambiente de la casa sino en prácticamente todos los salones y habitaciones. Había estantes llenos en la sala principal, en el estudio, en la salita de estar, en la biblioteca del primer piso; en el segundo nivel, en su dormitorio; y, sobre todo, en todas las habitaciones del sótano. La casa en sí reflejaba claramente el trabajo académico que su dueña y única habitante realizaba diariamente.

Recuerdo que la sala principal tenía estantes del suelo al techo con cientos de libros de estudios clásicos. Particularmente quedé impresionado con la enorme colección de autores grecolatinos de la editora Loeb, así como otros muchos textos especializados en el cristianismo temprano. Los libros de Historia de América Latina y de Historia Moderna Europea estaban principalmente en el sótano, aunque también había muchos en otras salas del primer piso. En los dormitorios se encontraban sobre todo novelas y otros libros de carácter literario. Aunque se trataba en su gran mayoría de libros modernos, había también impresos coloniales y otros textos antiguos impresos en Europa en latín y griego. La colección estaba compuesta principalmente por libros en inglés, pero también por muchos textos en otros idiomas como el castellano, alemán, francés, portugués y otros. Comprobé allí que la tarea que me había sido encomendada era ciertamente titánica.

Como dije, estando hospedado en Notre Dame, tenía que ir todos los días a la antigua casa de Sabine por ocho horas, incluidos sábados y domingos, con un equipo de estudiantes de esta misma universidad. Habitualmente yo me quedaba solo a completar tareas cuatro horas más. Tuve mucha suerte en trabajar con este equipo de jóvenes, seleccionados hábil y generosamente por el profesor Dressing, que en esto prestó también una invaluable ayuda. Juntos, realizamos el primer inventario detallado de la colección ingresando manualmente la información en computadoras y, en el caso de libros modernos que tenían códigos de barras, con aplicaciones específicas de catalogación que facilitaban mucho la tarea. Al mismo 
tiempo, procedimos a embalar, etiquetar y numerar los libros en cajas. ¡Llegamos a 268 cajas, que contenían cerca de 12500 libros!

La cuestión misma del traslado de la biblioteca fue una labor monumental, toda una odisea. Las cajas debían ser transportadas miles de kilómetros al sur, hacia el Perú. Con la ayuda de una empresa especializada en transporte de carga a nivel global, nuestro valioso y monumental encargo salió del puerto de Norfolk en Virginia recién el lunes 9 de septiembre de 2013. Su llegada estimada a El Callao sería el 28 de septiembre. Para tener una idea del volumen de carga, al llegar a Arequipa los trabajadores de nuestra universidad se tardaron unas cuatro horas en descargar y transportar las cajas desde el camión hasta las instalaciones de almacenamiento temporal de la biblioteca.

Mientras tanto, por acuerdo de los obispos de Sur Andino (los prelados de Ayaviri, Juli y Sicuani), el Instituto de Pastoral Andina había ya decidido otorgar la colección en comodato a la Universidad Católica San Pablo de Arequipa, que, por su condición académica, podía asumir los significativos costos de traslado, inventario, embalaje, embarque, trámites aduaneros y adecuadas medidas bibliotecarias, con el fin de custodiar la colección, ponerla en valor y ofrecerla a disposición del público académico nacional. Se procedió a la fumigación, clasificación, catalogación y posterior colocación en la biblioteca de la UCSP, donde ya desde un par de años atrás puede ser consultada. Hoy en día es una de las mayores colecciones bibliográficas de estudios andinos y de estudios clásicos en el periodo helenístico de nuestro país.

El profesor César Félix Sánchez Martínez, autor de un artículo conexo a estos breves apuntes en este mismo número y con quien tuve la suerte de dialogar estas breves reflexiones, me preguntaba qué era lo que unía tan profundamente a Sabine con el mundo andino. Me es difícil responder tales preguntas, pues no tuve la suerte de conocerla personalmente. Sin embargo, sí tuve la oportunidad de conversar con varias personas que la conocieron desde hace muchos 
años y desde varios ángulos diferentes. Tal parece que ella sentía una gran empatía por el mundo andino. No solo por la fascinación que sentía ante la grandeza de los incas sino por los hombres andinos de hoy, de alguna manera postergados y marginados. Viajó varias veces al Perú en estancias de investigación financiadas por varias instituciones académicas como Dumbarton Oaks. Allí tuvo ocasión de conocer el Instituto de Pastoral Andina del Cusco. Estaba suscrita a revistas de corte sociocultural que la vinculaban al mundo andino y, de hecho, en más de una ocasión colaboró económicamente con algunas iniciativas sociales en el Perú. Era muy generosa. Sé también que mantenía amistad con algunos colegas cusqueños, con quienes compartía conocimientos y a quienes ayudó en sus logros académicos, como fue el caso de Donato Amado quien llegó a Notre Dame por sus gestiones.

Por otro lado, pienso que profesionalmente era atractivo para ella ingresar en el mundo andino, pues las conexiones de tal universo con la cultura clásica grecolatina eran abundantes aunque poco estudiadas. Además, creo que Sabine vio en la conquista, y en el proceso de transformación religiosa que le siguió, un proceso de conversión religiosa similar al ocurrido en el periodo evangelizador temprano del Occidente cristiano, tema que ella conocía muy bien. Siendo ya una eminencia en los estudios clásicos en los Estados Unidos, decidió dar un giro en su carrera y abocarse a los estudios de la religión andina en el periodo hispánico. Definitivamente a ella le gustaban los retos. Conocí a una becaria en la UND que la tuvo como asesora y que fue estimulada a reorientar radicalmente su tema de tesis, pasando de los estudios sobre Perú a Mesoamérica. Sabine era de grandes desafíos. Trabajaba incansablemente todos los días. Según sus amigos, se levantaba a las 3 de la mañana para abocarse a sus investigaciones hasta el fin de la tarde.

Finalmente, me gustaría agradecer a Catherine MacCormack, su hija, y a Tom Cornfield, el albacea, quienes siempre tuvieron gran celo en hacer cumplir la voluntad de la donante. Esto no siempre 
fue fácil para ellos. Debían recorrer muchas millas para llegar a la casa de Sabine en South Bend y resolver los asuntos relativos a la donación. Les agradezco la confianza que tuvieron en mí y el apoyo que me dieron cuando llegué. Quisiera mencionar también a Bill Sparks, quien cuidaba de la casa y que me ayudó mucho con sus consejos prácticos y datos bastante útiles para realizar mi encargo.

Una de las personas que más me ayudaron fue David Dressing, quien me orientó mucho en el periodo previo al viaje, contactó al grupo de estudiantes y me introdujo al interesantísimo mundo de una biblioteca de investigación universitaria. A él, mil gracias. Agradezco a los chicos - hoy ya graduados- que trabajaron muy duro en el inventario y embalaje: Everaldo Aguiar, Pilar Giannini, Cristián Sailer, Justin McDevitt, Nga Le, entre otros.

Ya en el Perú, no puedo dejar de mencionar la gran disposición de las autoridades de la Universidad Católica San Pablo, comenzando por nuestro rector, Germán Chávez; de Miguel Salazar, prorrector para la Persona y la Cultura; de Alonso Quintanilla, nuestro vicerrector; Alejandra Maldonado, encargada de Relaciones Internacionales; y de Alejandro Estenós Loayza, entonces director de Investigación. También nos prestaron indispensable ayuda nuestros colegas Patricia Vela y Enrique Briceño, de la biblioteca de la UCSP; Héctor Cruz, asesor legal; así como Lía Chirinos y Pamela Cabala, de la Dirección de Investigación y del Centro de Estudios Peruanos de la UCSP, respectivamente.

Finalmente, nuestro agradecimiento se extiende especialmente a los obispos que conforman el directorio del Instituto de Pastoral Andina con sede en Cusco: a monseñor Miguel La Fay, entonces obispo de Sicuani; a monseñor Kay Schmalhausen, obispo de Ayaviri; y a monseñor José María Ortega Trinidad, obispo de Juli. Especial consideración debemos todos tener con las gestiones iniciales de nuestro amigo y colega Carlos Zegarra Moretti, secretario general de esta revista académica.

A todos ellos, nuestro más sentido agradecimiento y cariño. 